\title{
Measurement Framework for Analysis of Dynamic Behavior of Single-Phase Power Electronic Devices
}

\author{
Elias Kaufhold, Jan Meyer, Peter Schegner \\ Institute of Electrical Power Systems and High Voltage Engineering \\ Technische Universitaet Dresden \\ Dresden Germany \\ e-mail: elias.kaufhold@tu-dresden.de
}

\begin{abstract}
This paper studies the impact factors on the dynamic behavior of single-phase power electronic devices in low-voltage grids. White-box models are able to reflect the dynamic behavior, but require a detailed knowledge of the device. Black-box models can be easily parametrized by measurements, but are limited to small signal studies in frequency domain. No studies exist on black-box approaches reflecting the dynamic behavior of power electronic devices. A systematic, measurement based identification method considering the dynamic behavior is required to develop such dynamic black-box models. The aim of this paper is, to provide an overview of the impact factors on the dynamic behavior of power electronic devices as basis for the development of respective measurement procedures. An identification approach and its possible implementation for a laboratory test stand is proposed. Quantifying the impact factors with respect to the dynamic system response in terms of linear and non-linear characteristic provides the opportunity to develop a set of new dynamic models that can be used to improve e.g. stability studies for low voltage networks with a large penetration of modern power electronic devices in the future. First results are presented exemplarily for photovoltaic inverters.
\end{abstract}

Key words. black-box modeling, dynamic behavior, harmonic analysis, power electronics, power quality.

\section{Introduction}

Pursuing the climate goals leads to a growth of renewable energies as well as power electronic devices with topologies of higher energy efficiency. Wind power farms and photovoltaic systems have shown the largest increase over the last years [1]. The high demand results in a large diversity of commercially available photovoltaic (PV) systems and power electronics regarding their topology and parameters, especially for low-power applications in the low voltage grid [2]. The component that connects an application to the power grid is typically a power electronic device [3]. In this study, the term device refers to inverters, converters and rectifiers. Depending on the topology of such a device, the grid-side current can vary regarding its harmonic spectrum.

The study of the interaction between the power electronic device and the power grid has become a challenging research topic and received a large interest [4]. The output voltage of the device is typically pulsed, but smoothed by a grid-side filter. The amplitudes and phase angles of individual frequency components are important for the system behavior, e.g. with regard to resonances. These resonances occur because of interactions of the device and the power grid. The large diversity of the grid impedance in the low voltage grid [5] is a main challenge for prediction, analysis and simulation of the behavior of gridconnected devices. Another recent study objective is the assessment of the grid stability. Since the details about the design of power electronic devices are often not known and not disclosed by manufacturers, white-box modeling approaches cannot be used. While the implementation effort can be reduced, following a modular approach [6], the modeled device needs to be known in detail. Furthermore, white-box model simulations cause a high computational effort, so that the number of devices that can be studied within one simulation is very limited. Consequently black-box models are required that can fulfill the requirements of large-scale grid studies.

An advantage of black-box models is the reduced computational effort compared to white-box models. This is, because the details are missing and an aggregated behavior of all internal components is presented. The degree of complexity of these black-box models can vary. Known black-box models have considered constant current sources while being adapted later to current sources with impedances in terms of uncoupled Norton models and finally as state of the art developed into coupled Norton models. Both Norton models consist of a harmonic current vector and a respective harmonic admittance matrix. These black-box models and their parameters have to be identified based on appropriate measurements.

As state of the art, black box models are only applicable for small signal analysis, which reflects only steady state behavior. The operating point (steady state conditions) depends only on the voltage at the grid-side terminals of the device, which is defined in frequency domain by the amplitude and the phase angle of its frequency components. If no change occurs, the voltage at the grid-side terminals will stay constant in frequency domain, hence the gridimpedance and the background distortion do not have to be considered in steady state. A commonly used measurement 
procedure to identify the steady state behavior of inverters is the fingerprint method [7]. A frequency sweep is performed over the applied voltage at the grid-side terminals of the device and the current response is measured. By applying a discrete Fourier transform (DFT) on the measured current, an admittance matrix can be calculated with respect to the operating point. A stability analysis can be performed in frequency domain with impedance based stability analysis methods, e.g. according to the Nyquist criterion [3]. However, black-box models are up to now not applicable to model the dynamic behavior between two different steady states. At present, no systematic measurement procedure is known with respect to the study of the transient behavior of power electronic devices, e.g. for dynamic black-box modeling. First measurements show a strongly varying transient response on the same step change (e.g. in voltage distortion), even for different devices of one category.

The aim of this paper is to develop a framework as prerequisite to develop dynamic black-box models, namely a system of impacts factors to be considered for the measurement based quantification of the transient response of single-phase power electronic devices. The impact factors have to be classified with respect to the type of device (device category), e.g. inverters of photovoltaic systems. Each impact factor has to be evaluated with regard to its importance. If an impact factor has only a negligible impact for a specific device category, it does not have to be considered in the measurement-based identification for that category and the effort can be reduced. The quantification of the impact factors is determined by systematic laboratory measurements and forms the basis for the development of respective models.

The paper is structured as follows: Section II introduces the impact factors. In Section III, the test procedure as well as the evaluation of the measurements is proposed. An application example for commercially available photovoltaic inverters is given in Section IV and Section V concludes the proposed study and refers to future work.

\section{Impact factors}

To identify the impact factors, the general system model has to be studied in terms of interactions between a device and the power grid first. This general system model consists usually of two components: the power grid (grid-side) and the connected application with the power electronic device (device-side) as can be seen in Fig. 1. The device is considered the system component that connects a specific application to the power grid.

Based on this system model representation, the impact factors that can affect the interaction of the power grid with the power electronic device can also be categorized into impact factors of the power grid and impact factors of the power electronic device. The identified impact factors can be held constant or they are changed to trigger a transient response. At least one has to change to trigger a dynamic response.

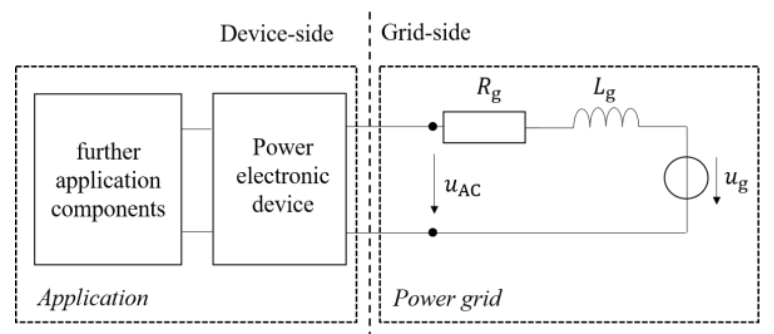

Fig. 1: System model

\section{1) Grid-side impact factors}

To understand the impact of the grid-side better, a brief description of the model of the power grid is given in the following. The model of the power grid consists typically only of passive components that reflect the aggregate of components of the utility and components of other connected devices, e.g. generators and consumers. A typical representation of the power grid consists nowadays of an $R L$-topology, e.g. [8]. However, this simplification does not represent the true nature of the grid, but a simplified and aggregated model of all grid-components when comparing it to real measurements [5]. Neglecting capacities can lead to a misleading representation of resonances. Typically, power electronic devices that are connected to the grid behave non-linear. The nonlinear behavior has an impact on other grid-connected devices. This can lead to resonances and other effects. These interactions are nowadays aggregated in terms of an impedance characteristic of the grid and a background distortion.

The impact of the grid-side can be separated into the impact of the grid-side voltage and the impact of the grid-side impedance. The grid-side impact factors are usually applicable for all different device categories.

\section{a) Grid-side voltage}

For the grid-side voltage in terms of steady-state, the frequency (harmonic order), phase angle and amplitude are distinguished. For measuring the dynamic behavior, a (step) change of the three impact factors above is applied, measured and evaluated. The amplitude and the phase angle of the added frequency component define the value of the jump in the reference signal. To define the time, when the step change is applied, another impact factor is required, namely the point on wave (PoW) with regard to fundamental voltage. A switching at the maximum will affect the maximum current that is injected into the grid. The PoW of the fundamental in terms of the switching moment can have an impact e.g. for phase-fired controllers.

With the increase of power electronics in the grid, it can be of interest to study preventively the reaction on abnormal conditions. Therefore, also the change at power frequency itself has to be considered. This includes phase jumps due to faults as well as changes in the amplitude in terms of voltage sags and swells. The proposed methodology is developed to study the frequency range above power frequency, hence subharmonics and DC-impacts are not further included in terms of the grid-side. The proposed tests in this study are based on the assumption, that the principle of superposition is applicable for further model development. This assumption has to be proven right for all 
tested device categories in an additional test. Also, for each device needs to be tested, if a pre-distortion will have an impact on the dynamic behavior.

\section{b) Grid-impedance}

Stationary analysis assumes that the grid impedance does not have an impact in steady state, if the system is stable. Nevertheless, the grid impedance defines the slew rate of the voltage and the current response in case of changes in the supply voltage and consequently also impacts the transient response. Next to the resonance frequency, the shape of the impedance at the resonance, namely its amplification is of importance for the transient response. For a test impedance in terms of laboratory tests, a topology that introduces a parallel resonance is suggested. A noncanonical $R L C$-topology can fulfill this requirement. Disconnecting the capacitor will result in a line characteristic of the impedance without any resonance that can be shifted by a variable $L$ and $R$. Connecting the $C$ will introduce the resonance while the resonance frequency can be adapted by changing the parameters for $L$ and $R$.

Switching the impedance will also trigger a transient response of the tested device. For changing the grid impedance, a categorization into fast changes and slow changes can be made. Slow changes will not trigger a transient response and are therefore not considered in this study. Fast changes on the other hand occur due to switching of components and changing operating points of grid-connected devices. A fast change will lead to a changing voltage drop over a grid impedance and hence cause a step change in the grid-side voltage at the inverter terminals. However, the reaction on voltage steps on the grid-side has been tested before. Considering passive impedances for laboratory implementation purposes, the impedance itself does not change after being switched. Therefore, the impedance does not need to be switched for the identification, since it is the same result as if the impedance would have existed as well before the step change.

\section{2) Device-side impact factors}

In order to specify the impact factors, a basic understanding of the functionality of the devices of each category is important since power electronic devices can show a large variety of topologies. While rectifiers can consist of a very simple structure, other devices, like inverters or converters can be significantly more complex. A proper categorization of devices is required in order to develop relevant impact factors and measurement procedures for characterizing their dynamic behavior. The structure of more complex power electronic devices can be divided into hardware and software components. Hardware components consist of circuit elements like resistors, inductances, switches, diodes etc., while the software components refer to the implemented control algorithms that affect typically the switching within the power electronic device. Even within one device category, such as PV-inverters, the devices can behave very differently [2]. The dynamic behavior of the current can vary strongly with respect to time constants and spectrum depending on the make of the device. In general, most components of the connected system will have an impact on the linear or nonlinear behavior of the device. All available components and their parameters that can be changed are possible impact factors. As a result, the deviceside impact factors can vary for each type of studied device.

As an example, the application defines, if the power level is expected to change or if it stays rather constant. For pumps with a fixed mechanical load and thus a fixed power, the power level will rather stay constant. Under this condition, the whole device-side impact can be neglected and the power level can be set constant with respect to typical operating points. However, there are applications where the power level changes significantly, e.g. the variation of the solar irradiance for PV-systems or the State of Charge (SOC) of electric vehicle chargers. Then, possible device-side impacts have to be studied as the transient response to power level changes as well as changes of other impact factors at different power levels can be of importance. Nevertheless, if a decoupling of the device-side with respect to the different time constants can be assumed, the DC-side impact is rather important for steady state analysis than for the dynamic behavior.

\section{3) Summary of impact factors}

A structured overview of all considered impact factors is given in the table below. This set does not claim to be complete and can even be extended if needed.

TABLE I. OVERVIEW OF IMPACT FACTORS

\begin{tabular}{|c|c|c|c|}
\hline & Impact f & tor & \\
\hline \multirow{10}{*}{ Grid-side } & \multirow{8}{*}{ Voltage } & \multirow{3}{*}{$\begin{array}{l}\text { Power } \\
\text { frequency }\end{array}$} & Point on wave \\
\hline & & & Phase jumps \\
\hline & & & Amplitude \\
\hline & & \multirow{5}{*}{ Harmonics } & Frequency \\
\hline & & & Amplitude \\
\hline & & & Phase angle \\
\hline & & & Superposition \\
\hline & & & Pre-distortion \\
\hline & \multirow[t]{2}{*}{ Impedance } & \multicolumn{2}{|c|}{ Resonance frequency } \\
\hline & & \multicolumn{2}{|c|}{ Resonance amplitude } \\
\hline Device-side & \multicolumn{3}{|c|}{ available component parameters } \\
\hline
\end{tabular}

\section{Test procedure and Evaluation}

While the individual impact factors have been introduced in the last section, the procedure to analyze these impact factors is explained in the following. For each step change (measuring point) any combination of the impact factors (number of frequency components, frequency, amplitude and phase angle of each component, point on wave of step change) is theoretically possible, which emphasizes the need for reducing the number of measuring points to a feasible amount. Therefore, firstly only the relevant impact factors for a specific device category have to be identified and in a second step only the relevant impact factors and their combinations have to be measured in more detail. For testing the impact of the grid-side voltage at this stage, the tests are distinguished into single frequency component 
tests and multiple frequency component tests. For single frequency testing, only the step change of one frequency component is applied to the reference signal, while for the multiple frequency testing a step change of multiple frequency components is applied.

\section{a) Single frequency component test}

For the single-frequency component tests, a non-distorted voltage is defined and applied as reference. Next a step change from the reference point to respective combinations of the impact factors is performed and the transient current response is recorded. This procedure is repeated for each measuring point.

The following impacts are tested based on single frequency testing: The PoW is varied with respect to the fundamental frequency, e.g. in $30^{\circ}$ steps as well as the phase angle of the added frequency component. Depending on the angle of the harmonic, the change will cause a jump in the reference at the time when it is added or come in smoothly. It also needs to be considered, if the frequency of the applied single frequency component has an impact on the transient response as well as the amplitude of the applied distorted single frequency component. For the frequency, according to the principle of harmonics, multiples of the fundamental frequency up to $2 \mathrm{kHz}$ are proposed with amplitude changes at a fixed value for all frequencies, e.g. $10 \mathrm{~V}$. In case of certain impact factors like pre-distortion the use of values based on related standards (e.g. compatibility level or immunity test standards) might be useful. Applying fixed magnitudes is more suitable for the development of models and a weak-point analysis.

A further test with respect to grouped frequencies considers the impact of a pre-distortion, i.e. the impact of the reference. In case the dynamic behavior is independent on the pre-distortion, the single frequency components do not need to be tested for other than the non-distorted reference point. For the test, it is proposed to use a typical voltage waveform of the public low-voltage grid, the "flat-top" [9], before the change is applied instead of the non-distorted sinusoidal voltage. To create a jump in the reference signal, the phase angle of a harmonic that is already present in the pre-distortion can be changed from $180^{\circ}$ or $0^{\circ}$ to $90^{\circ}$.

\section{b) Multiple frequency components test}

A second sub-category of testing considers grouped frequency components. It is proposed, to test the superposition principle with a distortion that consists of three frequency components. For single-phase devices, the power flow is often intermittent, so that a DC-capacitor buffers the energy in case of switched power electronic devices with an AC-DC conversion. This can lead to a sideband component of twice the fundamental frequency on the capacitor that can propagate into the grid and might be seen in sideband frequency couplings. It is therefore proposed to choose the 5th, 7 th and 9 th order harmonics for testing the superposition principle. In addition a comparison of a grouped frequency change with the three previously performed single frequency changes of the individual harmonics has to be performed to analyze the applicability of superposition.

\section{c) Summary of test procedures}

An overview of the previously described test procedures with respect to their application on the grid-side voltage at the terminals of the power electronic device is given in the table below.

TABLE II. TEST PROCEDURES FOR GRID-SIDE VOLTAGE

\begin{tabular}{|l|l|l|}
\hline \multirow{4}{*}{ Test procedure } & \multicolumn{2}{|l|}{ Grid-side voltage } \\
\hline \multirow{4}{*}{$\begin{array}{l}\text { Single frequency } \\
\text { component test }\end{array}$} & Point on wave & Fundamental \\
\cline { 2 - 2 } & Phase jumps & \\
\cline { 2 - 2 } & Amplitude & \\
\cline { 2 - 2 } & Frequency & \multirow{2}{*}{ Harmonics } \\
\cline { 2 - 2 } & Amplitude & \\
\cline { 2 - 2 } & Phase angle & \\
\cline { 2 - 2 } $\begin{array}{l}\text { Multiple frequency } \\
\text { component test }\end{array}$ & Pre-distortion & \\
\hline
\end{tabular}

\section{d) Evaluation of results}

For the evaluation of the dynamic behavior, three analysis indices are defined and evaluated for each measuring point: the overshoot, the eigenfrequencies and the settling time. For the dependency of the indices on the impact factors, three conditions can be distinguished: independent, linear dependent and non-linear dependent. If the indices are independent, the respective impact factor can be neglected. In case of a linear dependency, three different values for the impact factor should be measured. For non-linear relationships, multiple values are required, depending on the degree of nonlinearity.

\section{Application on photovoltaic inverters}

As the interactions with regard to the device significantly depend on the device category, in this study, single-phase low-power PV-inverters are used as example category. They are very common and a large variety of makes can be found in the market. To understand the interaction of PVinverters with the power grid, the operating principle of $\mathrm{PV}$-inverters is introduced shortly in the following.

\section{a) Operating principle}

$\mathrm{PV}$-inverters are the component in a PV-system that connect the DC-side (PV panels) to the AC-side (power grid). The structure of PV-inverters can be categorized into hardware and software components as shown in Fig. 2. Hardware components of the PV-inverter are typically a grid-side filter, the semi-conductor switches, a DC-link capacitor and typically a DCDC-converter to connect the DC-side (PV-Panels) to the inverter. The switching frequency of the switches will affect the minimum reaction time of the control. At the same time, the higher frequency distortion of an inverter is determined by the switching frequency. In some topologies, a High-frequency (HF)transformer and a grid-side Low-frequency (LF)transformer can be found. 


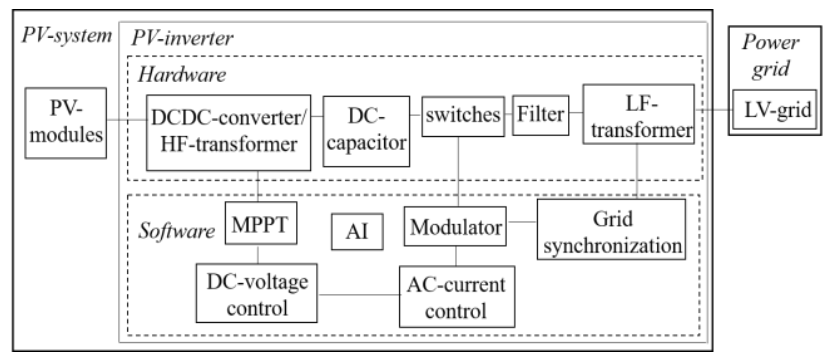

Fig. 2: PV-system with power grid

For the software components, the grid-synchronization is important for the transient response of the PV-inverter. Typically, this is a Phase locked loop (PLL), which determines the phase angle and the amplitude of the fundamental frequency of the voltage at the POC. Its bandwidth has an impact e.g. on the settling time of a transient response. Also, the time constants of the control can cause a delay in the transient response on step changes in the grid-side voltage. The modulator will generate the switching algorithm that leads to a pulsed voltage, which is smoothed by the grid-side filter circuit. A maximum power point tracker (MPPT) can optimize the operating point of the PV-system. The operating point of the DC-side, i.e. the DC-power level and the voltage at the DC-side inverter terminals $u_{\mathrm{DC}}$, can have a non-linear impact on the inverter behavior [10]. The identified MPP can be set e.g. via the DCDC-converter, which can generate harmonic emission itself. In addition, the MPPT will define the time constants of setting a new operating point and will consequently have an impact on the dynamic behavior of the PV-inverter. For specific circumstances, such as islanding, the manufacturers are required to implement respective detection algorithms, e.g. an Anti-Islanding (AI) detection. Sometimes, these algorithms can result in an unwanted behavior, such as tripping and therefore an unwanted shutdown of the PV-inverter. Voltage sags, phase jumps or a high distortion level can trigger this unwanted behavior [11].

For PV-inverters in low-power applications, the inverter is treated as an entire black-box, since the parameters of the control etc. can typically not be changed by the consumer. The only parameter that can be changed on the device side is the DC-power that results from the PV-panels.

\section{b) Measurement application}

In the following, an application of the proposed tests is presented exemplarily. In Fig. 3, measurements of the dynamic behavior of three different PV-inverters are shown. The voltage at the grid-side inverter terminals is changed in the maximum of the fundamental (PoW of $90^{\circ}$ ) adding a $250 \mathrm{~Hz}$ component at its maximum, so at a phase angle of $90^{\circ}$. The fundamental frequency component of the current of inverter 3 is lower, because inverter 3 is a threephase inverter consisting of three single-phase inverters but the measurements have been performed at the same DCpower level as for the single-phase inverters.

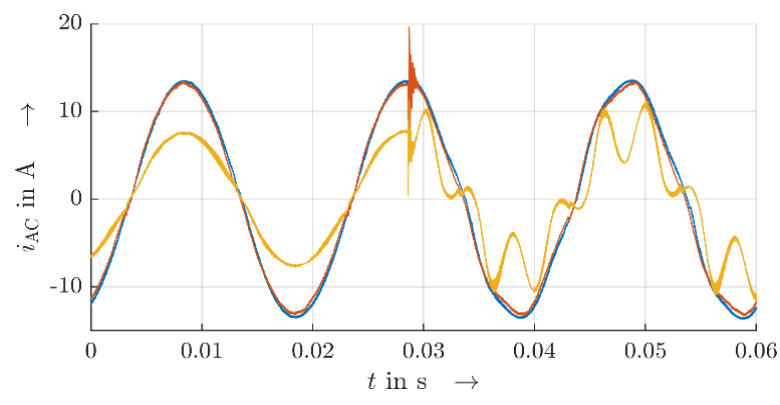

Fig. 3: Grid-side current of inverter 1 (blue), inverter 2 (red) and inverter 3 (yellow)

While inverter 3 shows a higher current distortion at the same applied steady state voltage at the inverter terminal, i.e. after 0.03 s, Fig. 4 depicts a zoomed part of Fig. 3 to show the dynamic behavior. It becomes visible, that even though the static behavior of inverter 3 is more critical, the dynamic behavior of inverter 2 shows an overshoot of about $9 \mathrm{~A}$, while the overshoot of inverter 3 is only about $6 \mathrm{~A}$, which underlines that steady-state and dynamic behavior are also qualitatively different between different inverters.

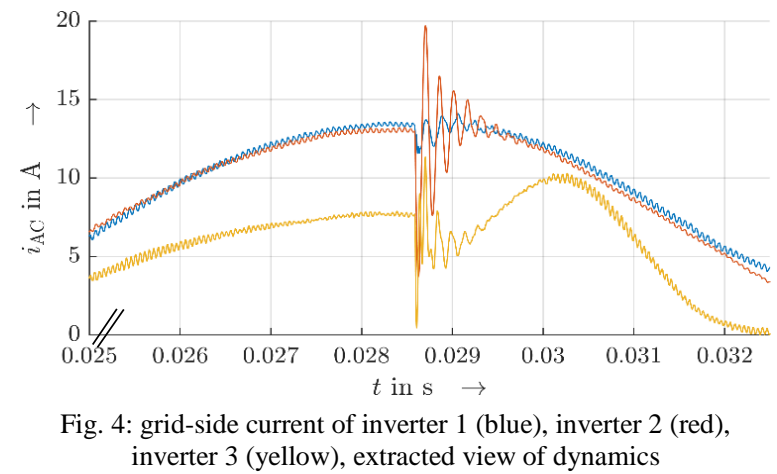

First measurements indicate that the transient response seems to be independent of the PoW for PV-inverters. Consequently, the identification procedure with respect to measuring points can be reduced.

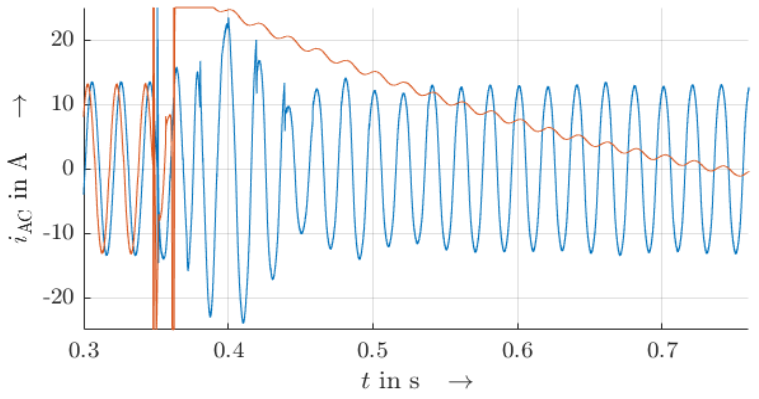

Fig. 5: PV-inverter 1 (blue) and PV-inverter 2 (red) at phase jump of fundamental frequency

Fig. 5 shows the different behavior of two PV-inverters under a $90^{\circ}$ phase jump in the fundamental frequency of the voltage at the grid-side inverter terminals. While this is a very large change, it can be seen, that inverter 2 is tripping, i.e. it shuts down, while inverter 1 remains in operation.

In Fig. 6, a current measurement is shown for inverter 2 at two different power levels and impedances. Impedances have been set to no impedance (noImp) and reference impedance according to IEC 60725 (Imp), while the background voltage distortion is changed. The power level is varied from half rated power (HP) to full rated power (FP) is applied. 


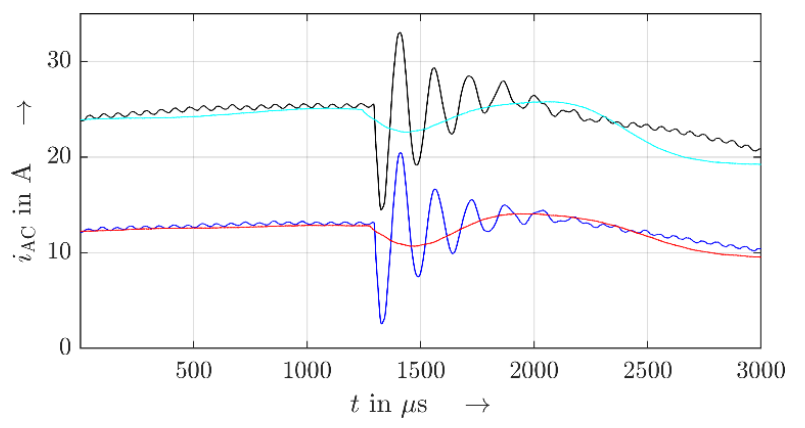

Fig. 6: $i_{\mathrm{AC} \text { noImp HP }}$ (blue), $i_{\mathrm{AC} \mathrm{Imp} \mathrm{HP}}\left(\right.$ red) $i_{\mathrm{AC} \text { nolmp FP }}$ (black), $i_{\mathrm{AC} \text { Imp FP }}$ (cyan)

While Fig. 6 shows the current, the voltage during the same transient process is shown in Fig. 7. It becomes visible, that the grid-impedance, even if not changed, has a significant impact on the dynamic behavior.

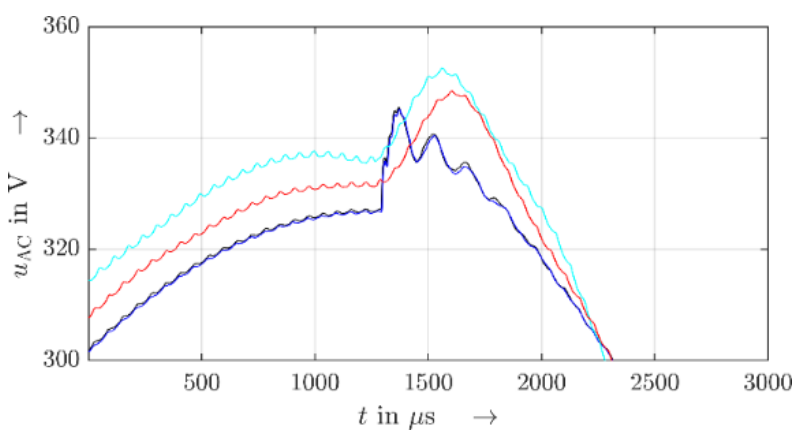

Fig. 7: $u_{\mathrm{AC} \text { nolmp HP}}$ (blue), $u_{\mathrm{AC} \text { Imp HP}}($ red $), u_{\mathrm{AC} \text { nolmp FP }}$ (black), $u_{\mathrm{AC}}$ Imp FP(cyan)

For PV-inverters, the DC-power level is seen to have an impact on the admittance characteristic, i.e. the stationary behavior that can affect the system stability [10]. For the dynamic behavior, first measurements seem to indicate, that the DC-power level is not of importance for the dynamic behavior, as can be seen in Fig. 6. If the time constants of MPPTs in PV-inverters are much larger than the dynamic process, the DC-side can be considered decoupled from the AC-side for photovoltaic inverters. This measurement has not been done yet to finally proof the decoupling for the studied PV-inverters.

\section{Conclusion}

The study presents a framework for the measurement-based characterization of the dynamic behavior of power electronic devices. The results of the measurements can be used to determine the "grid-robustness" of the devices, e.g. PV inverter, and can serve as prerequisite to develop new black-box modeling approaches to model the dynamic behavior for large scale simulations. Initial measurements for PV inverters have shown that the transient response is significantly different between different makes.

The slew rate of the step change is at the moment mainly determined by the grid-simulator in the laboratory, but the impact of the slew rate itself has to be studied in future work. The proposed impact factors are also relevant for three-phase systems but need some adaptation, such as considering balanced and unbalanced conditions. It is also left to future work to choose suitable models in time domain models, to fit the collected data into new models that can represent the dynamic behavior of power electronic devices in low-voltage grids.

\section{Acknowledgment}

This work was funded by the Deutsche Forschungsgemeinschaft (DFG, German Research Foundation) - 360497354.

\section{References}

[1] Blaabjerg, F. ; Yang, Y. ; Yang, D. ; Wang, X. Distributed Power-Generation Systems and Protection. In: Proceedings of the IEEE Bd. 105, Institute of Electrical and Electronics Engineers (IEEE) (2017), Nr. 7, S. 1311-1331.

[2] Teodorescu, R. ; Liserre, M. ; Rodriguez, P.: Grid Converters for Photovoltaic and Wind Power Systems : Wiley-IEEE Press, 2011 - ISBN 9780470057513.

[3] Sun, J.: Impedance-Based Stability Criterion for GridConnected Inverters. In: IEEE Transactions on Power Electronics Bd. 26, Institute of Electrical and Electronics Engineers (IEEE) (2011), Nr. 11, S. 3075-3078.

[4] Wang, X. ; Blaabjerg, F.: Harmonic Stability in Power Electronic Based Power Systems: Concept, Modeling, and Analysis. In: IEEE Transactions on Smart Grid, Institute of Electrical and Electronics Engineers (IEEE) (2018), S. 1.

[5] Stiegler, R. ; Meyer, J. ; Schori, S. ; Höckel, M. ; Drápela J. ; Hanzlík, T.: Survey of network impedance in the frequency range 2-9 $\mathrm{KHZ}$ in public low voltage networks in AT / CH / CZ / GE. In: 25th International Conference on Electricity Distribution, 2019, S. 3-6.

[6] Kaufhold, E. ; Meyer, J. ; Schegner, P.: Modular WhiteBox Model of single-phase Photovoltaic Systems for Harmonic Studies. In: 13th IEEE PES PowerTech Conference. Milano, Italy, 2019.

[7] Cobben, S. ; Kling, W. ; Myrzik, J.: The Making and Purpose of Harmonic Fingerprints. In: 19th International Conference on Electricity Distribution (2007), Nr. 0764, S. 21-24.

[8] Liserre, M. ; Teodorescu, R. ; Blaabjerg, F.: Stability of photovoltaic and wind turbine grid-connected inverters for a large set of grid impedance values. In: IEEE Transactions on Power Electronics Bd. 21, Institute of Electrical and Electronics Engineers (IEEE) (2006), Nr. 1, S. 263-272.

[9] Langella, R. ; Testa, A. ; Meyer, J. ; Moller, F. ; Stiegler, R. ; Djokic, S.Z.: Experimental-Based Evaluation of PV Inverter Harmonic and Interharmonic Distortion Due to Different Operating Conditions. In: IEEE Transactions on Instrumentation and Measurement Bd. 65 (2016), Nr. 10, S. 2221-2233 - ISBN 9781479909483.

[10] Kaufhold, E. ; Meyer, J. ; Müller, S. ; Schegner, P.: Probabilistic Stability Analysis for Commercial Low Power Inverters Based on Measured Grid Impedances. In: 2019 9th International Conference on Power and Energy Systems. Perth, Australia, 2019.

[11] Enslin, J.H.R. ; Heskes, P.J.M.: Harmonic Interaction Between a Large Number of Distributed Power Inverters and the Distribution Network. In: IEEE Transactions on Power Electronics Bd. 19, Institute of Electrical and Electronics Engineers (IEEE) (2004), Nr. 6, S. 15861593. 